\title{
ITPI: Initial Transcription Process-Based Identification Method of Bioactive Components in Traditional Chinese Medicine Formula
}

\author{
Baixia Zhang, ${ }^{1}$ Yanwen $\mathrm{Li}^{2}{ }^{2}$ Yanling $\mathrm{Zhang}^{1}{ }^{\mathrm{Zhiyong}} \mathrm{Li},{ }^{3} \mathrm{Tian} \mathrm{Bi}^{3}$ \\ Yusu He, ${ }^{1}$ Kuokui Song, ${ }^{3}$ and Yun Wang ${ }^{1}$ \\ ${ }^{1}$ School of Chinese Pharmacy, Beijing University of Chinese Medicine, Beijing 100102, China \\ ${ }^{2}$ Institute of Information on Traditional Chinese Medicine of China, Academy of Chinese Medical Sciences, Beijing 100700, China \\ ${ }^{3}$ China Minority Traditional Medical Center, Minzu University of China, Beijing 100081, China
}

Correspondence should be addressed to Zhiyong Li; lizhiyong7899@163.com and Yun Wang; wangyun@bucm.edu.cn

Received 25 August 2015; Revised 9 February 2016; Accepted 11 February 2016

Academic Editor: Kuzhuvelil B. Harikumar

Copyright (C) 2016 Baixia Zhang et al. This is an open access article distributed under the Creative Commons Attribution License, which permits unrestricted use, distribution, and reproduction in any medium, provided the original work is properly cited.

\begin{abstract}
Identification of bioactive components is an important area of research in traditional Chinese medicine (TCM) formula. The reported identification methods only consider the interaction between the components and the target proteins, which is not sufficient to explain the influence of TCM on the gene expression. Here, we propose the Initial Transcription Process-based Identification (ITPI) method for the discovery of bioactive components that influence transcription factors (TFs). In this method, genome-wide chip detection technology was used to identify differentially expressed genes (DEGs). The TFs of DEGs were derived from GeneCards. The components influencing the TFs were derived from STITCH. The bioactive components in the formula were identified by evaluating the molecular similarity between the components in formula and the components that influence the TF of DEGs. Using the formula of Tian-Zhu-San (TZS) as an example, the reliability and limitation of ITPI were examined and 16 bioactive components that influence TFs were identified.
\end{abstract}

\section{Introduction}

Identification of the bioactive components present in a traditional Chinese medicine (TCM) formula using computational method not only is fast and efficient, but also has the prospect of explaining the mechanism of action. The main identification methods reported to date are computer virtual screening technology [1], network pharmacology [2], directed TCM grammar systems (dTGS) [3], system pharmacology [4], and so forth. These methods mainly consider the effect of the components that are present in a TCM formula on the expressed proteins and do not sufficiently explain why a TCM formula affects the expression of a number of proteins. Here, we propose a new method, the Initial Transcription Process-based Identification (ITPI) method, to characterize the bioactive components present in a TCM formula. This method identifies the bioactive components by considering the potential interaction between the components and the transcription factors (TFs) of differentially expressed genes (DEGs). When used in combination with the previously reported methods, the ITPI method can not only help explain the mechanism of action, but also enable a more comprehensive discovery of the bioactive components that are present in a formula.

\section{Methods and Materials}

2.1. Principle of ITPI. Previous studies have shown that TCM formulas can influence gene expression $[5,6]$. However, the reported methods only consider the influence of the TCM on expressed proteins and fail to explain the role of the TCM on gene expression. Transcription is an important event during gene expression. TFs are key players in the transcription and are also drug targets $[7,8]$, but they are usually ignored. 


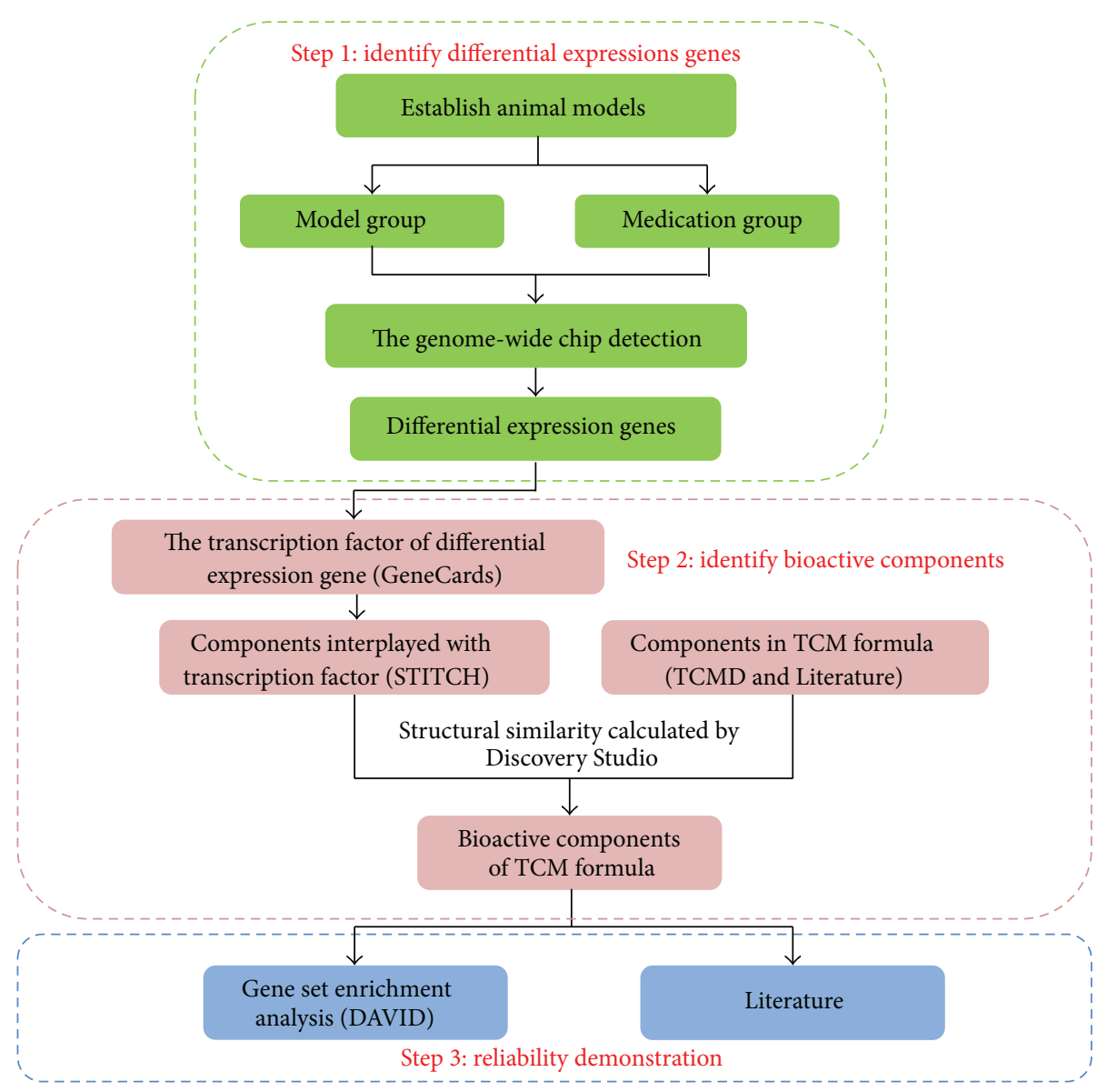

FIGURE 1: The workflow of ITPI.

TFs may be the targets of a given TCM formula, and the interactions of the formula with TFs may be an important mechanism by which the TCM formula exerts its biological effect. Therefore, the present study established the ITPI method to characterize the bioactive components present in a TCM formula. Here, we also discuss each step of the ITPI method (Figure 1) in detail.

The expression level of each gene in the medication and model groups is detected by the genome-wide chip detection technology. The normalized data are expressed as means \pm standard deviation (SD). Comparisons between the medication and model groups are then performed by one-way analysis of variance (ANOVA), followed by Student's $t$-test. Values of $P<0.05$ is considered statistically significant, and genes with significant expression levels are regarded to be DEGs.

With the development of bioinformatics and chemoinformatics, many TF databases $[9,10]$ and the component-protein interaction databases [11] have been established. Most of these databases contain the data on various organisms, including human, rat, and mouse. Therefore, care should be taken in choosing an organism with regard to the specific purpose of the investigation. In the ITPI method, the most relevant TFs of DEGs are derived from GeneCards (http://www.genecards .org/), and the potential components that can interact with the TF are derived from the STITCH (http://stitch.embl.de/).
In STITCH, the potential components that can interact with TFs are identified by name or SMILES string. The required confidence is higher than 0.400 , and the active prediction methods include gene fusion, cooccurrence, coexpression, experiments, databases, neighborhood, predictions, and text mining.

To date, many studies on the components of TCM formulas have been carried out and a large number of components have been separated and identified. The known components of a given TCM formula can be derived from the literature, which is included in China National Knowledge Internet (CNKI), PubMed (1979 2015), and the traditional Chinese medicines database (TCMD) [12]. The name, structure, and the SMILES of the known components should be recorded. Finally, repetition of the name of the components, both in the literature and in TCMD, is eliminated. When the component exists as a synonym, the repeated component name is identified by structure, and these instances are eliminated.

Molecular similarity is an important theme in chemoinformatics [13]. Its use is based on the principle that compounds with similar structures exhibit similar chemical properties and biological activities [14]. Although molecular similarity can also be calculated using molecular properties, such as $\log P$ and molecular weight, it is mostly defined as a distance measure in a structural or physicochemical descriptor 
space. There are many different distance measures available. These measures include Tanimoto, Dice, Cosine, and Soergel [15]. Concerning the fingerprint properties, the most popular similarity measure used for comparing the chemical structures represented by fingerprints is the Tanimoto (or Jaccard) coefficient $T$ [15]. Therefore, the Tanimoto coefficient was selected for the determination of molecular similarity. Two structures are usually considered to be similar if $T>0.85$. After the similarity calculation, the components that potentially interact with TFs can be determined. The Tanimoto coefficient is defined as follows:

$$
\text { Tanimoto coefficient }=\frac{\mathrm{SA}}{(\mathrm{SA}+\mathrm{SB}+\mathrm{SC})},
$$

where SA is the number of AND bits (bits present in both the target component and the reference component); SB is the number of bits present in the target component, but not in the reference component; and SC is the number of bits present in the reference component, but not in the target component.

Using this method, the bioactive components that are present in the TCM formula that can interact with TFs can be identified, indicating which bioactive components influence the expression of DEGs. The reported identification methods consider the interaction between the components and proteins expressed by DEGs, whereas ITPI identifies the bioactive components from the initial transcription process and regards TFs as drug targets. Combining other methods with ITPI, the bioactive components can be comprehensively discovered along the overall regulation process of TCM formula.

\subsection{Application of ITPI on TZS. Tian-Zhu-San (TZS), which consists of two Chinese medicinal herbs, Gastrodiae Rhizoma and Trillium, is mainly used for the treatment of neurodegen- erative diseases in the Tujia ethnic minority [16]. Gastrodiae Rhizoma is the tuber of Gastrodia elata BL. (Orchidaceae) and Trillium is the rhizome of Trillium tschonoskii Maxim. (Liliaceae). Although many studies have attempted to identify the bioactive components that are present in TZS [17-20], the component that acts against neurodegenerative diseases remains unknown. TZS can influence gene expression and likely interacts with TFs.}

2.2.1. Preparation of TZS Superfines. Gastrodiae Rhizoma and Trillium were purchased from the Anguo (Hebei Province). Dr. Yanwen Li of the Institute of Information on Traditional Chinese Medicine of China performed the identification and authentication of the samples. The dried herbs were ground into superfine powder (grain diameter $\leq 15 \mu \mathrm{m}$ ) using an Ultra-Micro Pulverizer. The superfine powder was dissolved in distilled water and stored at $4^{\circ} \mathrm{C}$ before its administration to rats.

2.2.2. Animals. Male Sprague-Dawley rats weighing within 190 219 g (Beijing Vital River Laboratory Animal Technology Co. Ltd.) were used for in vivo experiments. The animals were housed at $21 \pm 2^{\circ} \mathrm{C}$ under a 12-hour light/12-hour dark cycle (lights on at 07:00), were allowed to acclimatize, and were provided free access to water and food for extra week. All animal care and experimental procedures were performed in accordance with the National Institutes of Health Guide for the Care and Use of Laboratory Animals. The procedures were approved by the Committee on Animal Care of the Beijing University of Chinese Medicine. All efforts were made to minimize animals suffering and to reduce the number of animals used in the experiments.

\subsubsection{Model Preparation of Vascular Dementia Induced by} Chronic Cerebral Hypoperfusion and Drug Delivery. All procedures were performed according to the reported methods [21]. The animals were injected with $10 \%$ chloral hydrate $\left(0.35 \mathrm{~g} \cdot \mathrm{Kg}^{-1}\right)$ intraperitoneally. After administration of anesthesia, the animals were placed in a supine position and a median incision of approximately 1.5 to $2 \mathrm{~cm}$ was made on the neck. The bilateral common carotid arteries were isolated and ligated, and the incision was sutured. The animals were fed with the same diet for 60 days under the same conditions (Statement: our laboratory has established a stable method to duplicate the model of vascular dementia induced by chronic cerebral hypoperfusion. Based on the research purposes, this study omitted the neurobehavioral evaluation section).

The surviving rats were randomly divided into model and medication groups. After 24 hours, $625 \mathrm{mg} \cdot \mathrm{Kg}^{-1}$ superfine powder of TZS (the effective dose was calculated by the method of body weight) was administered to the medication group, and the blank and model groups were administered distilled water. This intragastric administration was continued for 60 days in all groups.

2.2.4. The Genome-Wide Chip Detection. The hippocampal tissues of the medication and model group rats were taken and stored in liquid nitrogen. Total RNA was extracted by the Trizol method and then purificated. The RNA quality of RNA was assessed by agarose gel electrophoresis. Then, using the purified total RNA, double stranded cDNA was synthesized, which was then fragmented and fluorescently labeled.

Fragmented cDNA and the reference reagents were then mixed, and the hybridization solution was prepared. The hybridization solution was poured onto an Affymetrix GeneChip Rat Gene 2.0ST Array. After 16 hours of hybridization, the chip was removed from the Hybridization Oven 645. With the adding of eluent and staining fluid, the wash and staining were completed in fluidics station 450 .

The microarray results were scanned using a GeneChip Scanner 3000, and the original data were read using the Command ConsoleSoftware 3.1. After normalizing the data, the signal strength of the medication group and model groups was compared. If the ratio was $>2$, the gene was considered to be the significantly upregulated expression gene, and if the ratio was $<0.5$, the gene was considered to be significantly downregulated expression gene.

2.2.5. Discover the Bioactive Components of TZS for Antivascular Dementia. Analyses of the gene expression data, the TFs of the DEGs, the components that can interact with the TFs of the DEGs, the components of the TCM formula, and the molecular similarity were all carried out according to the 
principles of ITPI. It should be noted that when components existed as synonyms, we deleted the repeated component names, using the "ChemBioFinder for Office 12.0." Molecular similarity was calculated using the "Find Similar Molecules by Fingerprints" module of the Discovery Studio 4.0 [22].

\subsection{Validation of Identification Results. To demonstrate the} rationality of ITPI, we performed the following: verifying the relationship between the pharmacological action of the bioactive components and vascular dementia by looking up literature and the other was verifying whether the DEGs which were regulated by bioactive components related to vascular dementia by gene set enrichment analysis [23]. GeneGO term enrichment analysis was performed to highlight the most relevant biological pathways associated with a given gene list. DAVID 6.7 Functional Annotation Clustering was used for this purpose. $P<0.05$ indicated significantly enriched biological pathways.

\section{Results and Discussions}

3.1. Bioactive Components of TZS for Antivascular Dementia. After normalizing the results from genome-wide chip detection, we compared the medication group and model groups (Supplemental Information 1 in Supplementary Material available online at http://dx.doi.org/10.1155/2016/8250323) and found 229 DEGs, which included 219 upregulated genes $(>2)$ and 10 downregulated genes $(<0.5)$ (Supplemental Information 2). The TFs of the DEGs (Supplemental Information 3 ) and the components that interacted with the TFs (Supplemental Information 4) are listed in the Supplementary Material. A total of 52 components (Supplemental Information 5) of TZS were used to calculate the molecular similarity. The results of the molecular similarity calculations revealed that there are 16 components whose Tanimoto coefficients were greater than 0.85 and involved 13 TFs and 30 DEGs (Table 1).

These results showed that one component can regulate several TFs or genes; different components can regulate the same gene on account of their interaction with the same TF; that is to say, one gene or TF can be affected by multiple components. This indicates that multiple bioactive components of TZS interplay with multiple genes or TFs and produce the antivascular dementia effect.

3.2. Validation of Identification Results. 30 DEGs were used for the enrichment analysis, and 10 DEGs were significantly enriched in 7 clusters (Table 2). The pathogenesis of vascular dementia mainly involves the cholinergic system, inflammatory processes, oxygen free radicals, and the transport of NO, among others [24-26]. These enriched biopathways, such as blood circulation [27], oxidation reduction [28], hormone metabolic process [29], and oxygen transport [30], were all found to be closely related to vascular dementia. That is to say, the DEGs of Hba-a2, F5, Nts, Cyp11b3, Cyp2j4, Cyp2a2, $\mathrm{Nqo}$, $\mathrm{Ttr}, \mathrm{Ngf}$, and $\mathrm{Hbb}$ were also found to be closely related to vascular dementia.

The results of enrichment analysis showed that 10 of the 30 DEGs were closely related to vascular dementia. These 10
DEGs were found to be regulated by 16 components. This indicated that 16 components were indeed effective against vascular dementia and that TZS acts against antivascular dementia mainly by regulating these 10 DEGs (Table 1). Gene set enrichment analysis that is based on the DEGs is a commonly used method. The enrichment analysis, which was based on the 30 DEGs regulated by the bioactive components, was more focused and specific.

A literature search can be used to identify some of the vascular dementia-related pharmacological effects of the components. For example, it has reported that bis(4-hydroxybenzyl)ether exerts a neuroprotective effect in an ischemic model [31], $\beta$-sitosterol protects neural stem cells from neurodegenerative diseases [32], diosgenin has an anti-inflammatory effect and acts against oxidative during the monocrotalineinduced pulmonary hypertension in rats $[33,34]$, and daucosterol induces a protective Th1 immune response against disseminated Candidiasis in mice [35]. Although there is no direct proof of the interaction between the components and the respective TFs or genes, the studies described above are indicative of the rationality of using ITPI for the identification of bioactive components to some extent.

\section{Conclusion}

Using the newly developed ITPI method, we identified 16 components of TZS and 10 DEGs that are closely related to vascular dementia. The results demonstrated the utilizing of ITPI in the identification of bioactive components and its rationality. The ITPI method focuses on the overall components of a given TCM formula and genes of treatment objects, along with the initial transcription process of "all of the components of TCM formula-TFs-DEGs." Compared with the reported methods, such as computer virtual screening technology and network pharmacology, ITPI could not only identify the bioactive components but also help explain why a given TCM formula influences the gene expression. The ITPI method also identifies the targeted TFs and genes of the bioactive components by combining the methods of technology of bioinformatics and cheminformatics. Meanwhile, the results suggest that a TCM formula exerts its therapeutic effect by regulating multiple TFs through the action of multiple components.

The ITPI method still has some limitations. Gene expression is controlled at multiple levels, including transcription, and TFs can regulate the initial process of transcription. Interaction with TFs may be only one of the many ways in which TCM exerts its biological effect, but its small impact could produce a huge change just because initial transcription process was the upstream of the biological pathway. Because the results were based on existing data included in databases, the genes in the similarity calculation only accounted for a portion of the total amount of DEGs; the data of TFs regulated DEGs remains to be perfect. Further studies on the TCM formula components, and the related TFs and genes, along with improved databases, will enable a more comprehensive analysis and detailed characterization of the bioactive components. The correlation coefficient and the action mode of the interplay between the components and the TFs were not 
TABLE 1: Sixteen bioactive components and their regulated TFs and DEGs.

\begin{tabular}{|c|c|c|c|c|c|}
\hline $\mathrm{TCM}$ & TZS components & $\begin{array}{c}\text { The CID of } \\
\text { component that } \\
\text { interacts with } \mathrm{TF}\end{array}$ & $\begin{array}{l}\text { Tanimoto } \\
\text { coefficient }\end{array}$ & $\mathrm{TF}$ & Differential expression genes \\
\hline \multirow{20}{*}{$\begin{array}{l}\text { Gastrodia elata } \\
\text { BL. }\end{array}$} & 4,4'-Dihydroxydiphenyl methane & 6623 & 0.877023 & ER-alpha & F5/Hba-a2/Rnasel11 \\
\hline & \multirow{2}{*}{$\beta$-Sitosterol } & 2371 & 0.915282 & Sp1 & Rnase4/F5/Slc19a3 \\
\hline & & 2524 & 0.896709 & Egr-1 & Ifitm 7 \\
\hline & Citric acid & 311 & 1 & Olf-1 & Сур2a2/Сyp2j4 \\
\hline & $\begin{array}{l}\text { 3-Hydroxy-3- } \\
\text { methoxycarbonylpentanedioic } \\
\text { acid }\end{array}$ & 311 & 0.8 & Olf-1 & Сур2a2/Сyp2j4 \\
\hline & \multirow{2}{*}{ Daucosterol } & 2371 & 0.879428 & Sp1 & Rnase4/F5/Slc19a3 \\
\hline & & 2524 & 0.852015 & Egr-1 & Ifitm7 \\
\hline & \multirow{3}{*}{ Margaric acid } & 985 & 0.994764 & Sp1 & Rnase4/F5/Slc19a3 \\
\hline & & 2506 & 0.941581 & Sp1 & Rnase4/F5/Slc19a3 \\
\hline & & 148177 & 0.875424 & Sp1 & Rnase4/F5/Slc19a3 \\
\hline & Bis(4-Hydroxybenzyl)ether & 6623 & 0.850932 & ER-alpha & F5/Hba-a2/Rnasel11 \\
\hline & \multirow[t]{2}{*}{ Vitamin A } & 5538 & 0.970093 & c-Fos & $\begin{array}{c}\text { Dusp12/Gpatch4/Ngf/Nts/Hba- } \\
\text { a2/RGD1564469 }\end{array}$ \\
\hline & & 1744 & 0.856672 & CHOP-10 & Ttr/Kcnj13 \\
\hline & \multirow{2}{*}{ Palmitic acid } & 985 & 1 & Sp1 & Rnase4/F5/Slc19a3 \\
\hline & & 2506 & 0.933009 & Sp1 & Rnase4/F5/Slc19a3 \\
\hline & \multirow{5}{*}{ Palmitoyl glycerol } & 2506 & 0.96445 & Sp1 & Rnase4/F5/Slc19a3 \\
\hline & & 985 & 0.94697 & Sp1 & Rnase4/F5/Slc19a3 \\
\hline & & 3987 & 0.916115 & Egr-1 & Ifitm7 \\
\hline & & 148177 & 0.884291 & Sp1 & Rnase4/F5/Slc19a3 \\
\hline & & 2499 & 0.852916 & STAT5B & Clic6 \\
\hline \multirow{18}{*}{$\begin{array}{l}\text { Trillium } \\
\text { tschonoskii } \\
\text { Maxim. }\end{array}$} & \multirow{3}{*}{ Pennogenin } & 2524 & 0.890232 & Egr-1 & Ifitm7 \\
\hline & & 2371 & 0.882143 & Sp1 & Rnase4/F5/Slc19a3 \\
\hline & & 54454 & 0.863784 & ATF-2 & Cyp2j4/Rasl11a/Slc16a13 \\
\hline & \multirow{3}{*}{ Linoleic acid } & 1424 & 0.932432 & RelA & Cyp2j4/RT1-T24-4/Nqo2 \\
\hline & & 3987 & 0.865169 & Egr-1 & Ifitm7 \\
\hline & & 1444 & 0.860317 & STAT3 & $\begin{array}{c}\text { Clic6/Hbb/Krt18/Fpr- } \\
\text { rs3/Ifitm7/RGD1564126/Zfp467 }\end{array}$ \\
\hline & 4-Hydroxybenzoic acid & 44540357 & 0.8679 & c-Jun & $\begin{array}{c}\text { Dusp12/Gpatch4/Ngf/Fpr- } \\
\text { rs3/Hba-a2/RGD1564469/S100vp }\end{array}$ \\
\hline & \multirow{2}{*}{$\begin{array}{l}\text { 3-O- } \alpha \text {-L-Rhamnopyranosyl }(1 \rightarrow 3) \\
\text { sterylglucoside }\end{array}$} & 2371 & 0.879428 & Sp1 & Rnase4/F5/Slc19a3 \\
\hline & & 2524 & 0.852015 & Egr-1 & Ifitm7 \\
\hline & \multirow{5}{*}{ 26-Chloro-26-deoxycryptogenin } & 2371 & 0.886708 & Sp1 & Rnase4/F5/Slc19a3 \\
\hline & & 2524 & 0.878365 & Egr-1 & Ifitm 7 \\
\hline & & 146898 & 0.862239 & STAT2 & Clic6/Naip5/Cyp11b3 \\
\hline & & 54454 & 0.855305 & ATF-2 & Cyp2j4/Rasl11a/Slc16a13 \\
\hline & & 400769 & 0.853801 & STAT3 & $\begin{array}{c}\text { Clic6/Hbb/Krt18/Fpr- } \\
\text { rs3/Ifitm7/RGD1564126/Zfp467 }\end{array}$ \\
\hline & \multirow{4}{*}{ Diosgenin } & 5753 & 0.853659 & FOXO3 & Fpr-rs3/RGD1559708 \\
\hline & & 2371 & 0.885714 & Sp1 & Rnase4/F5/Slc19a3 \\
\hline & & 2524 & 0.881 & Egr-1 & Ifitm7 \\
\hline & & 54454 & 0.866091 & ATF-2 & Cyp2j4/Rasl11a/Slc16a13 \\
\hline
\end{tabular}

The Tanimoto coefficient of these components was greater than 0.85 ; this table shows the corresponding relationship between the bioactive components, TFs and DEGs. 
TABLE 2: The significantly enriched biopathways.

\begin{tabular}{|c|c|c|c|c|}
\hline GO ID & Term & Count & $P$ value & Genes \\
\hline GO:0003013 & Circulatory system process & 4 & 0.001231 & Hba-a2/F5/Nts/Cyp11b3 \\
\hline GO:0008015 & Blood circulation & 4 & 0.001231 & Hba-a2/F5/Nts/Cyp11b3 \\
\hline GO:0055114 & Oxidation reduction & 5 & 0.009123 & Cyp2j4/F5/Cyp11b3/Cyp2a2/Nqo2 \\
\hline GO:0042445 & Hormone metabolic process & 3 & 0.010025 & Ttr/Cyp11b3/Ngf \\
\hline GO:0015671 & Oxygen transport & 2 & 0.016748 & $H b a-a 2 / H b b$ \\
\hline GO:0010817 & Regulation of hormone levels & 3 & 0.020049 & Ttr/Cyp11b3/Ngf \\
\hline GO:0015669 & Gas transport & 2 & 0.025023 & $H b a-a 2 / H b b$ \\
\hline
\end{tabular}

Values of $P<0.05$ were considered as significantly enriched biopathways.

considered in the present study. In addition, the way that compounds affect TFs is indirect or direct but unclear. Therefore, we could not describe the potency and the action mode of the TZS components that interplay with TFs and DEGs.

ITPI identifies bioactive components based on the TFs that they influence. The results will be more comprehensive if ITPI is used in tandem with other methods. In this study, we discovered that TZS cured vascular dementia by affecting blood circulation and oxygen transport and by regulating hormone levels. Our further goal is to draw a detailed biological network, describe the relationship between these bioactive components and biomolecules, and explain the mechanism by which the components of TZS act against vascular dementia at a molecular level. We can also carry out the drug repositioning by reversing the ITPI, screen the components that target specific genes or TFs associated with a disease, and find a specific drug combination for treating a given disease. The present study provides a novel platform for the identification of bioactive components present in a TCM formula, which can be applied more widely in the research of TCM formula studies.

\section{Conflict of Interests}

The authors declare that there is no conflict of interests.

\section{Acknowledgments}

This work was supported by the National Natural Science Foundation of China (Grant nos. 81001693, 81173568, 81373985, and 81473797), Young Talents Program of Beijing Municipal Commission of Education (Grant no. YETP1293), and Program for New Century Excellent Talents in University (Grant no. NCET-11-0605).

\section{References}

[1] X. Wang, Z. Z. Ren, Y. S. He, Y. Xiang, Y. Zhang, and Y. Qiao, "A combination of pharmacophore modeling, molecular docking and virtual screening for iNOS inhibitors from Chinese herbs," Bio-Medical Materials and Engineering, vol. 24, no. 1, pp. 13151322, 2014.

[2] X. Li, L. Wu, W. Liu et al., "A network pharmacology study of Chinese medicine QiShenYiQi to reveal its underlying multicompound, multi-target, multi-pathway mode of action," PLoS ONE, vol. 9, no. 5, Article ID e95004, 2014.
[3] J. Luo, Y. L. Ren, H. Gu, Y. Wu, and Y. Wang, "dTGS: method for effective components identification from traditional Chinese medicine formula and mechanism analysis," Evidence-Based Complementary and Alternative Medicine, vol. 2013, Article ID 840427, 9 pages, 2013.

[4] J. L. Liu, M. Q. Pei, C. L. Zheng et al., "A systems-pharmacology analysis of herbal medicines used in health improvement treatment: predicting potential new drugs and targets," EvidenceBased Complementary and Alternative Medicine, vol. 2013, Article ID 938764, 17 pages, 2013.

[5] Z.-J. Zhang, Z. Wang, X.-Y. Zhang, K. Ying, J.-X. Liu, and Y.-Y. Wang, "Gene expression profile induced by oral administration of baicalin and gardenin after focal brain ischemia in rats," Acta Pharmacologica Sinica, vol. 26, no. 3, pp. 307-314, 2005.

[6] Z. J. Zhang, P. Li, Z. Wang et al., "A comparative study on the individual and combined effects of baicalin and jasminoidin on focal cerebral ischemia-reperfusion injury," Brain Research, vol. 1123, no. 1, pp. 188-195, 2006.

[7] P. G. Richardson, C. Mitsiades, R. Schlossman et al., "Bortezomib in the front-line treatment of multiple myeloma," Expert Review of Anticancer Therapy, vol. 8, no. 7, pp. 1053-1072, 2008.

[8] K. Siddiquee, S. Zhang, W. C. Guida et al., "Selective chemical probe inhibitor of Stat3, identified through structure-based virtual screening, induces antitumor activity," Proceedings of the National Academy of Sciences of the United States of America, vol. 104, no. 18, pp. 7391-7396, 2007.

[9] W. Edgar, "The TRANSFAC project as an example of framework technology that supports the analysis of genomic regulation," Briefings in Bioinformatics, vol. 9, no. 4, pp. 326-332, 2008.

[10] M. Safran, I. Dalah, J. Alexander et al., "GeneCards version 3: the human gene integrator," Database (Oxford), vol. 2010, Article ID baq020, 2010.

[11] M. Kuhn, D. Szklarczyk, S. Pletscher-Frankild et al., "STITCH 4: integration of protein-chemical interactions with user data," Nucleic Acids Research, vol. 42, no. 1, pp. D401-D407, 2014.

[12] X. Zhou, S. Chen, B. Liu et al., "Development of traditional Chinese medicine clinical data warehouse for medical knowledge discovery and decision support," Artificial Intelligence in Medicine, vol. 48, no. 2-3, pp. 139-152, 2010.

[13] N. Nikolova and J. Jaworska, "Approaches to measure chemical similarity-a review," QSAR and Combinatorial Science, vol. 22, no. 9-10, pp. 1006-1026, 2004.

[14] G. M. Maggiora and V. Shanmugasundaram, "Molecular similarity measures," in Chemoinformatics and Computational Chemical Biology, vol. 672 of Methods in Molecular Biology: Methods and Protocols, pp. 39-100, Springer, Berlin, Germany, 2011. 
[15] D. Bajusz, A. Raca, and K. Heberger, "Why is Tanimoto index an appropriate choice for fingerprint-based similarity calculations," Journal of Cheminformatics, vol. 7, article 20, 2015.

[16] T. Bi, K. K. Song, W. X. Du, Z. Y. Li, Y. W. Li, and M. Zhang, "The therapeutic effect of Tianzhusan on dementia diseaselike incuced with D-galactose in mice," Lishizhen Medicine and Materia Medica Research, vol. 24, no. 11, pp. 2593-2595, 2013.

[17] X.-H. Duan, Z.-L. Li, D.-S. Yang, F.-L. Zhang, Q. Lin, and R. Dai, "Study on the chemical constituents of Gastrodia elata," Zhong Yao Cai, vol. 36, no. 10, pp. 1608-1611, 2013.

[18] X.-D. Yang, J. Zhu, R. Yang, J.-P. Liu, L. Li, and H.-B. Zhang, "Phenolic constituents from the rhizomes of Gastrodia elata," Natural Product Research, vol. 21, no. 2, pp. 180-186, 2007.

[19] X. Gao, W. Sun, Q. Fu, and X. Niu, "Rapid identification of steroidal saponins in Trillium Tschonoskii maxim by Ultraperformance Liquid Chromatography Coupled to Electrospray ionization quadrupole Time-of-Fight Tandem Mass Spectrometry," Phytochemical Analysis, vol. 26, no. 4, pp. 269-278, 2015.

[20] Z.-L. Zhang, Y.-M. Zuo, Y.-Y. Wang, M.-T. Cai, and Y.-Y. Li, "Study on chemical constituents in the roots and rhizome of Trillium tschonoskii (III)," Zhong Yao Cai, vol. 36, no. 11, pp. 1779-1782, 2013.

[21] H. Zhao, Z. Y. Li, Y. L. Wang, and Q. X. Zhang, "Hippocampal expression of synaptic structural proteins and phosphorylated cAMP response element-binding protein in a rat model of vascular dementia induced by chronic cerebral hypoperfusion," Neural Regeneration Research, vol. 7, no. 11, pp. 821-826, 2012.

[22] J. D. Holliday, N. Salim, M. Whittle, and P. Willett, "Analysis and display of the size dependence of chemical similarity coefficients," Journal of Chemical Information and Computer Sciences, vol. 43, no. 3, pp. 819-828, 2003.

[23] H. Huang, Y. Tang, W. He, Q. Huang, J. Zhong, and Z. Yang, "Key pathways and genes controlling the development and progression of clear cell renal cell carcinoma (ccRCC) based on gene set enrichment analysis," International Urology and Nephrology, vol. 46, no. 3, pp. 539-553, 2014.

[24] J. Wang, H.-Y. Zhang, and X.-C. Tang, "Cholinergic deficiency involved in vascular dementia: possible mechanism and strategy of treatment," Acta Pharmacologica Sinica, vol. 30, no. 7, pp. 879-888, 2009.

[25] H. Nagasawa and K. Kogure, "Multi-focal delayed neuronal damage after transient regional ischemia: mechanism of vascular dementia," Clinical Neurology, vol. 30, no. 4, pp. 396-401, 1990.

[26] Y. Yamasaki, N. Matsuura, H. Shozuhara et al., "Interleukin-1 as a pathogenetic mediator of ischemic brain damage in rats," Stroke, vol. 26, no. 4, pp. 676-681, 1995.

[27] M. Q. Wei, J. Z. Tian, J. Shi, F. Y. Ma, Y. C. Miao, and Y. Y. Wang, "Effects of Chinese medicine for promoting blood circulation and removing blood stasis in treating patients with mild to moderate vascular dementia: a randomized, double-blind and parallel-controlled trial," Zhong Xi Yi Jie He Xue Bao, vol. 10, no. 11, pp. 1240-1246, 2012.

[28] D.-H. Choi, K.-H. Lee, J.-H. Kim et al., "NADPH oxidase 1, a novel molecular source of ROS in hippocampal neuronal death in vascular dementia," Antioxidants and Redox Signaling, vol. 21, no. 4, pp. 533-550, 2014.

[29] M. Carantoni, G. Zuliani, M. R. Munari, K. D’Elia, E. Palmieri, and R. Fellin, "Alzheimer disease and vascular dementia: relationships with fasting glucose and insulin levels," Dementia and Geriatric Cognitive Disorders, vol. 11, no. 3, pp. 176-180, 2000.
[30] T. Zhang, Q.-W. Yang, S.-N. Wang et al., "Hyperbaric oxygen therapy improves neurogenesis and brain blood supply in piriform cortex in rats with vascular dementia," Brain Injury, vol. 24, no. 11, pp. 1350-1357, 2010.

[31] N.-K. Huang, Y. J. Chern, J.-M. Fang, C.-I. Lin, W.-P. Chen, and Y.-L. Lin, "Neuroprotective principles from Gastrodia elata," Journal of Natural Products, vol. 70, no. 4, pp. 571-574, 2007.

[32] A. Hamedi, A. Ghanbari, V. Saeidi, R. Razavipour, and H. Azari, "Effects of $\beta$-sitosterol oral administration on the proliferation and differentiation of neural stem cells," Journal of Functional Foods, vol. 8, no. 1, pp. 252-258, 2014.

[33] L. A. Ahmed, A. A. Z. Obaid, H. F. Zaki, and A. M. Agha, "Role of oxidative stress, inflammation, nitric oxide and transforming growth factor-beta in the protective effect of diosgenin in monocrotaline-induced pulmonary hypertension in rats," European Journal of Pharmacology, vol. 740, pp. 379-387, 2014.

[34] M. Singh, A. A. Hamid, A. K. Maurya et al., "Synthesis of diosgenin analogues as potential anti-inflammatory agents," Journal of Steroid Biochemistry and Molecular Biology, vol. 143, pp. 323-333, 2014.

[35] J.-H. Lee, J. Y. Lee, J. H. Park et al., "Immunoregulatory activity by daucosterol, a $\beta$-sitosterol glycoside, induces protective Th1 immune response against disseminated Candidiasis in mice," Vaccine, vol. 25, no. 19, pp. 3834-3840, 2007. 


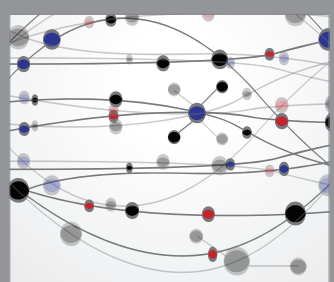

The Scientific World Journal
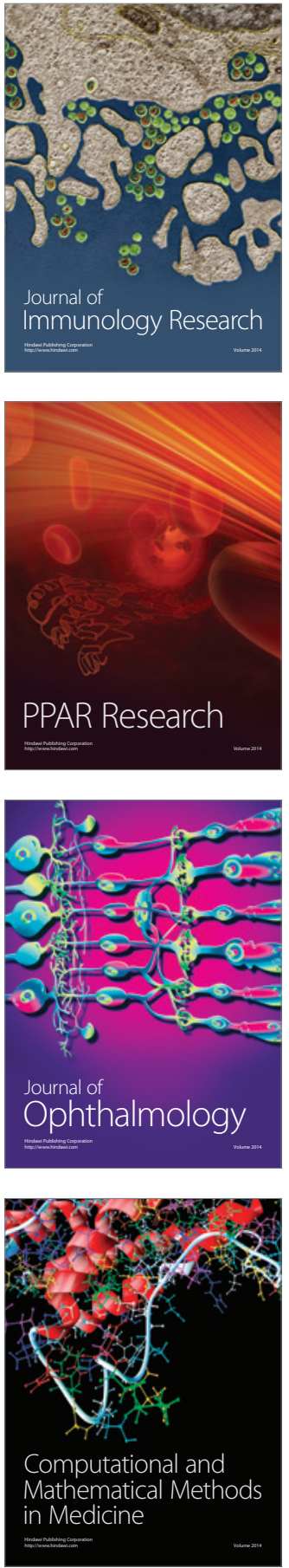

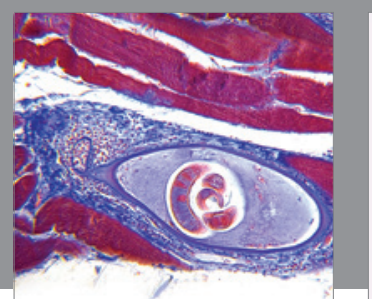

Gastroenterology Research and Practice

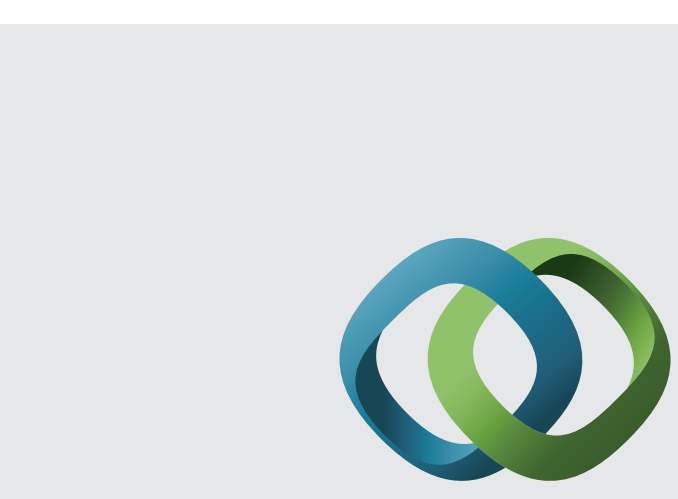

\section{Hindawi}

Submit your manuscripts at

http://www.hindawi.com
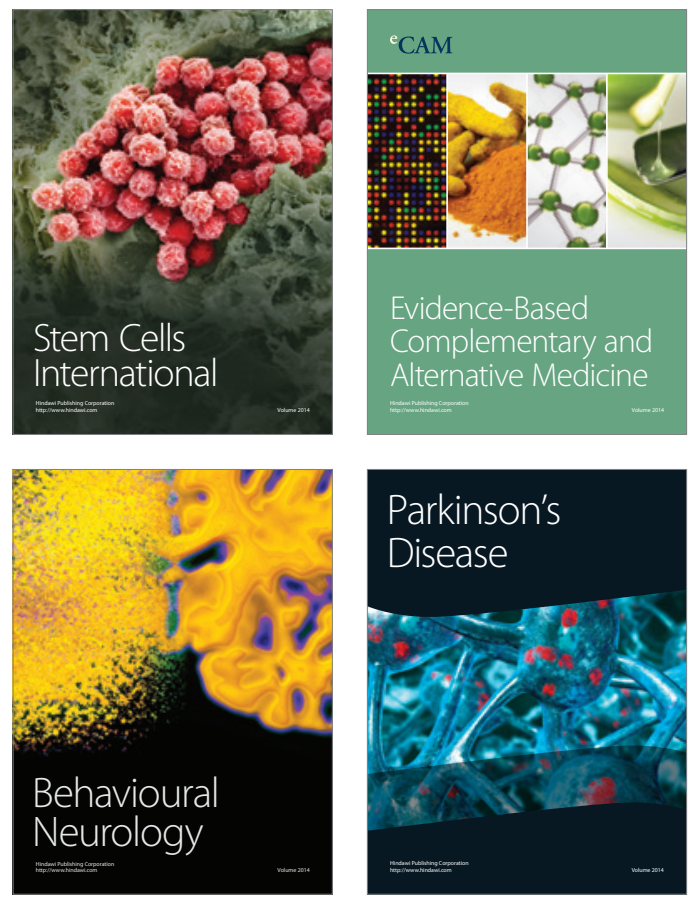
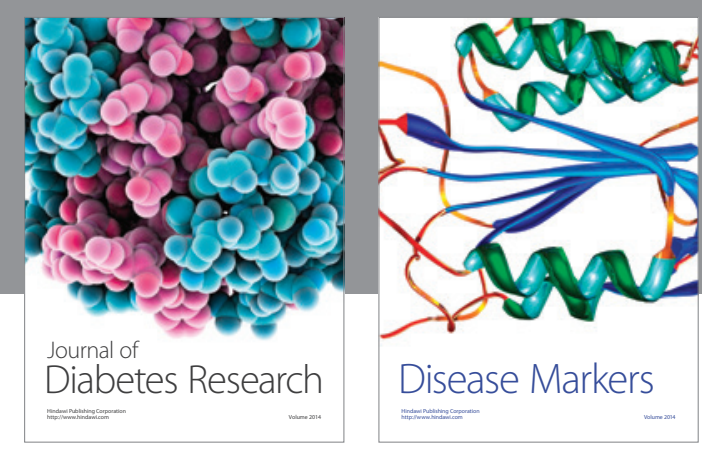

Disease Markers
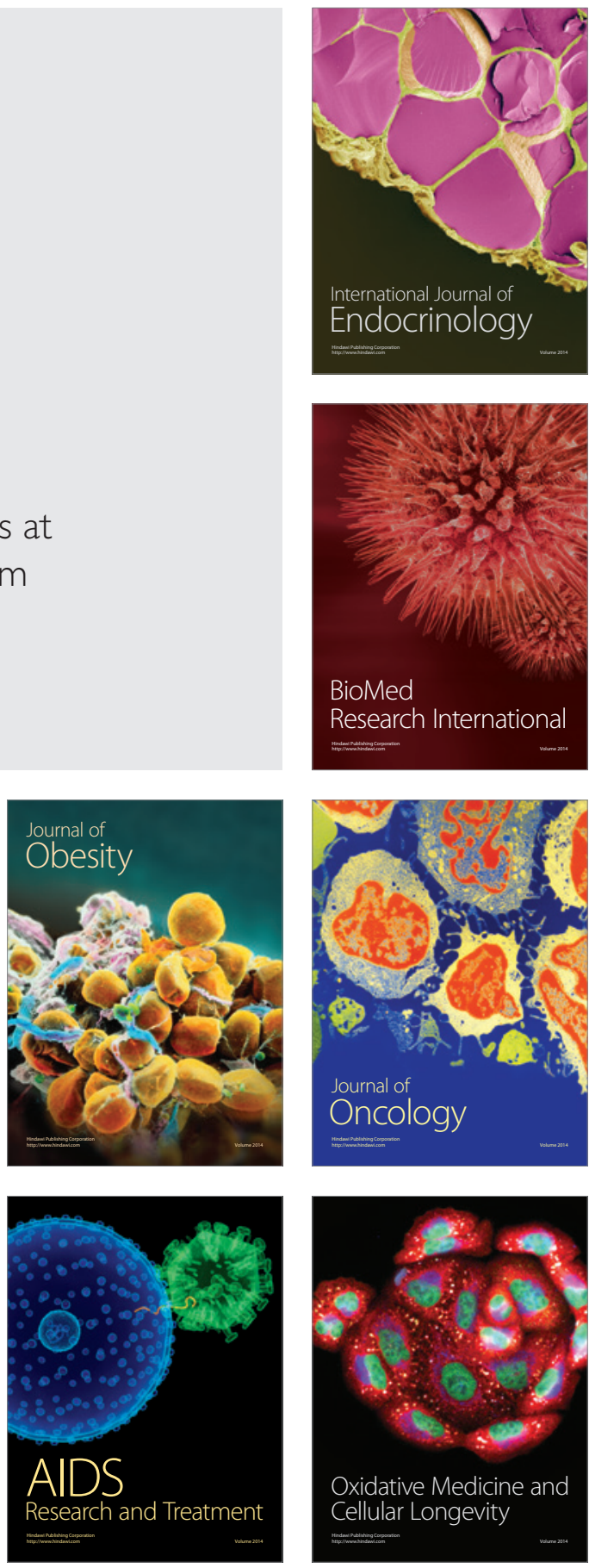